\title{
An Evaporative Coolant Used to ECR Ion Source
}

\author{
Lin RUAN ${ }^{1, a}$, Shun-Zhou $\mathrm{YU}^{2, \mathrm{~b}}$, Bin $\mathrm{XIONG}^{3, c}$ \\ ${ }^{1,2,3}$ Institute of Electrical Engineering, Chinese Academy of Sciences, Beijing, China \\ arosaline@mail.iee.ac.cn, ${ }^{\mathrm{b}}$ kezhi@mail.iee.ac.cn, ${ }^{\mathrm{c} x i o n g b i n @ m a i l . i e e . a c . c n}$
}

Keywords: Evaporative coolant, ECR ion source, Anti-irradiation, Stability.

\begin{abstract}
As a kind of high efficient cooling mode, the evaporative cooling technology has been successfully applied to a lot of engineering fields. This paper introduces a kind of evaporative coolant, for the first time, used to the ECR ion source. Firstly, we compared the basic physical and chemical characteristics of the coolant with F113; then, the anti-irradiation test was carried out for the coolant samples labeled with different time span, finally, the real demonstrating application of the coolant displayed good cooling effect and sound stability, which shows the selection of the coolant is correct and can be extended to use in various magnetic equipment.
\end{abstract}

\section{Introduction}

The evaporative cooling technology takes advantage of the physical process of phase change heat exchange to cool the heating parts. For different usage, it has different cooling structure [1]. Among that, the immersion self-circulation (ISC) is a kind of most efficient cooling structure. The principle of ISC evaporative cooling technology is on the basis of two-phase fluid dynamics theory and heat transfer theory [2], which has been applied in other fields of engineering technology, such as the boiler, the nuclear reactor and the transportation of oil and gas in pipeline. In our country, IEECAS has developed this technology for more than 50 years and successfully applied it to a lot of industry fields such as generators, motors, power transformer, electronics equipments and supercomputers, etc. for this technology, cooling medium, evaporative coolant, is the key issue. For different application which has different cooling target and different operation environment, we provide different coolant. This paper introduces a kind of evaporative coolant used to ECR ion source (ECRIS). Through qualitative analysis, experimental research, instrumental analysis and final demonstrating application in LECR4, we concluded that this evaporative coolant is the right selection for ECRIS and can be extended to use to any other kind of electromagnetic equipments among the whole accelerator.

\section{The Principle of ISC Evaporative Cooling ECRIS}

It is well known, the Heavy ion accelerator is very important scientific appliance used for the scientific research and medical therapy such as nuclear physics, atomic physics, tumor therapy research, and other heavy ion applications, etc. Ion source as its component is the most important part should to be well designed and maintained. Typically, for the room temperature ECR ion source, magnets and solenoids are cooled with de-ionized pressured-water and it is very costly if the excitation current density reaching above $10 \mathrm{~A} / \mathrm{mm} 2$ and magnet power approaching a few hundred kilowatts. Avoiding the disadvantages of the water cooling [3], we put forward a kind of ISC evaporative cooling method for ECRIS [4]. The operational principle (shown in Fig. 1) is as follows: the solenoids and magnets are totally immersed in the liquid-phase evaporative coolant, the magnets forms the main magnetic path and at the same time function as the part of tank for the evaporative coolant. When the coolant inside absorb the heats from the solenoids and magnets, its temperature will rise gradually, when the temperature reaches the boiling point, the liquid-phase coolant will become gas-phase coolant and absorb large quantity of heat so as to cooling the magnets and solenoids. Based on the vertical cooling structure arrangement, the gas-phase coolant will rise to condenser and transfer the heat to the water to be condensed to liquid-phase coolant and flow back to 
the tank, next circulation start. From it circulation, we can see that it is self-circulation with no external momentum. For this cooling structure, the coolant has direct contact with the solenoid and magnets made of electrical pure iron. The coolant not only plays a role of cooling, but also it must have the good insulation and material stability.

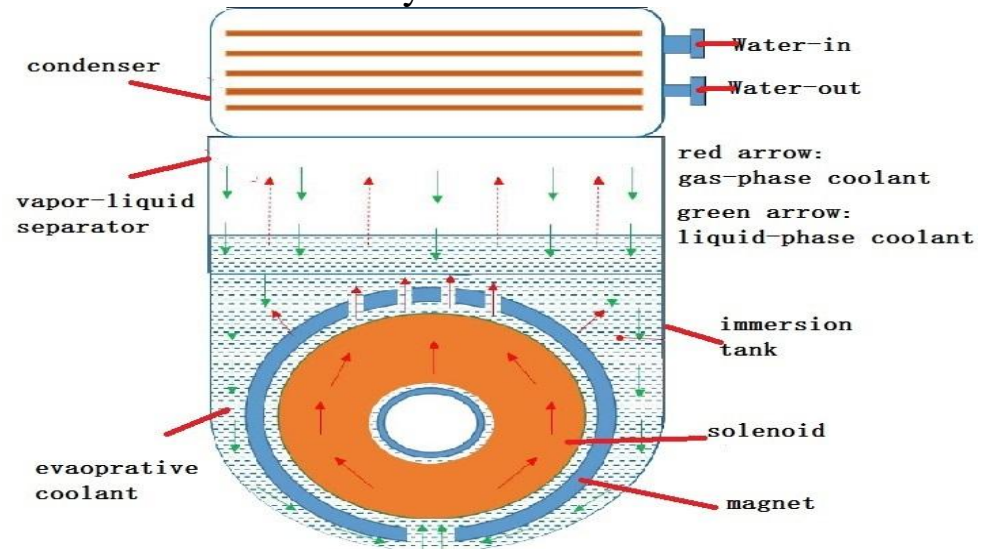

Fig.1 the operational principle of ISC evaporative cooling system

\section{The Selection of Coolant}

Evaporative cooling and water cooling are all belong to liquid cooling method, but they use different cooling medium. Just because the evaporative coolant is with high insulation, so it is safer than water. In the Evaporative cooling system, the choice of cooling medium is very important. Besides the good insulation properties, the medium used in the cooling system should meet the following requirements: appropriate evaporation temperature and a larger latent heat; good flow properties; good chemical stability; good compatibility; safe and non-toxic and not combustion. Based on our previous fundamental research and successful application cases, we accumulated a lot of experiences on coolant selection and built up a library of evaporative coolants with the boiling temperature range from $30-80^{\circ} \mathrm{C}$. Considering the above requirements, $\mathrm{F} 113$ is relatively optimum choice for cooling electrical equipment. But it would destroy the ozonosphere when it rises to the stratosphere. On the basis of the Montreal protocol, after the year of 2006, our country as developing country must stop to use it. So, we have already chosen some new environmentally friendly insulating coolants to substitute for the primary F113 in order to ensure the sustainable development of the evaporative cooling technology. Accord to the cooling target of ECRIS, the temperature of the sextupole permanent magnet cannot exceed $50^{\circ} \mathrm{C}$ and the temperature of solenoid below $65^{\circ} \mathrm{C}$, comprehensive analysis of the heat transfer performance and the design of whole system, we recommend the EC-A-1 (our internal series number) as the working coolant for the ECRIS. From the following comparison, we can see that the performance of EC-A-1 is very near and to somehow better than F113 especially the environmental index.

Tab. 1 Comparison of characteristics between F113 and EC-A-1

\begin{tabular}{|c|c|c|}
\hline & EC-A-1 & CFC-113 \\
\hline Boiling point $\left({ }^{\circ} \mathrm{C}\right)$ & 47 & 47.6 \\
\hline Liquid density $(\mathrm{g} / \mathrm{cm} 3)$ & 1.62 & 1.57 \\
\hline Latent heat cal/g) & 29.37 & 35.6 \\
\hline Solution of water $(\mathrm{ppm})$ & 42 & 110 \\
\hline $\begin{array}{c}\text { withstand voltage }(\mathrm{kV})(2.5 \mathrm{~mm} \\
\text { gap) }\end{array}$ & $>20$ & $>20$ \\
\hline ODP & 0 & 0.8 \\
\hline GWP & 64 & 5000 \\
\hline
\end{tabular}

Note: latent heat is at boiling temperature, others at $25^{\circ} \mathrm{C}$. 


\section{The Anti-irradiation Analysis of the Coolant}

From the aspect of materials contacting directly with the coolant, the application of the evaporative coolant in ECRIS has no differences with the other application such as generators except for the operational environment. There exist high energy rays such as X-ray near the ECRIS. So we specially carried out the test of the coolant in the irradiation condition. We put our testing coolant by the side of ECRIS during its operation for a certain time, then take out sample labeled with different time period for instrumental analysis. The instrument we used is the Agilent 7890 liquid chromatograph. The analysis results (Fig.2) show that the content of the coolant has changed to some extent. It because that the transition of isomer happened. But the two isomers are similar on the physical and chemical performance, so it will not affect the performance of coolant and long-time use. At the same time, one month sample has no difference with the 2 weeks sample, so time span effect can be neglected. In one word, after analysis, the coolant can be long-term used in such irradiation environment and safe for the ECRIS.

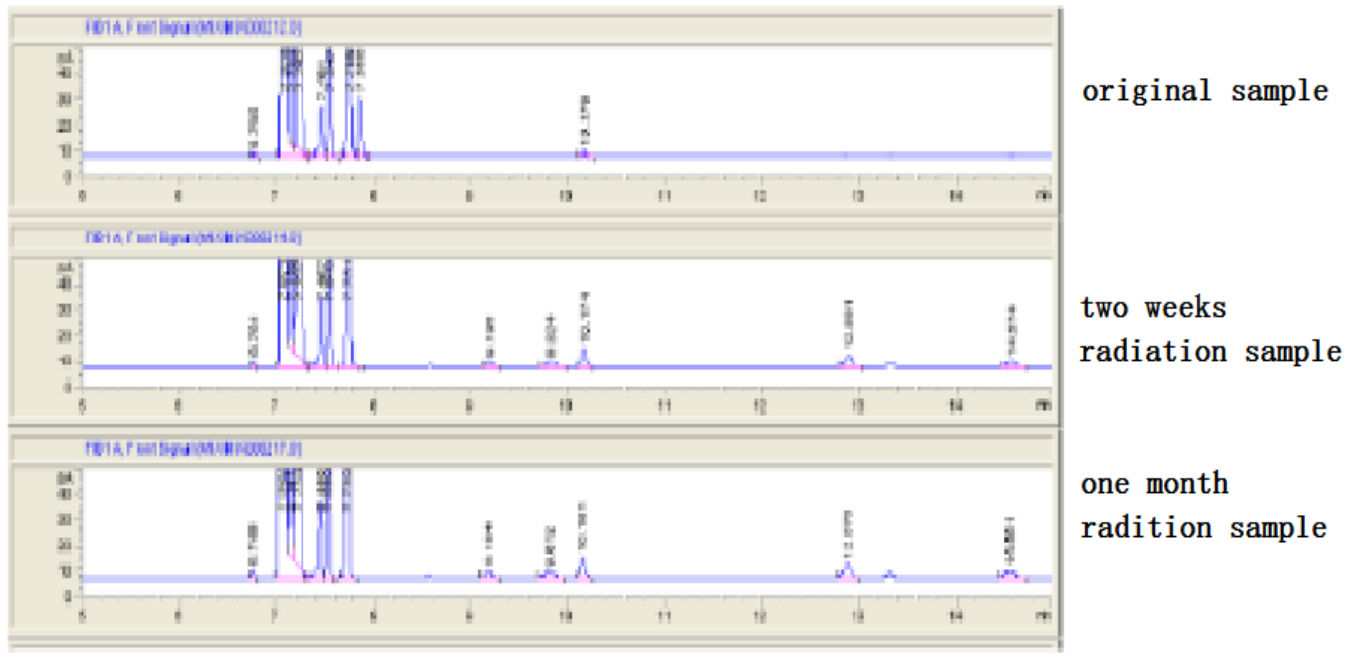

Fig. 2 The chromatograph analysis results of the coolant after different radiation periods

\section{The Demonstrating Application of the Coolant}

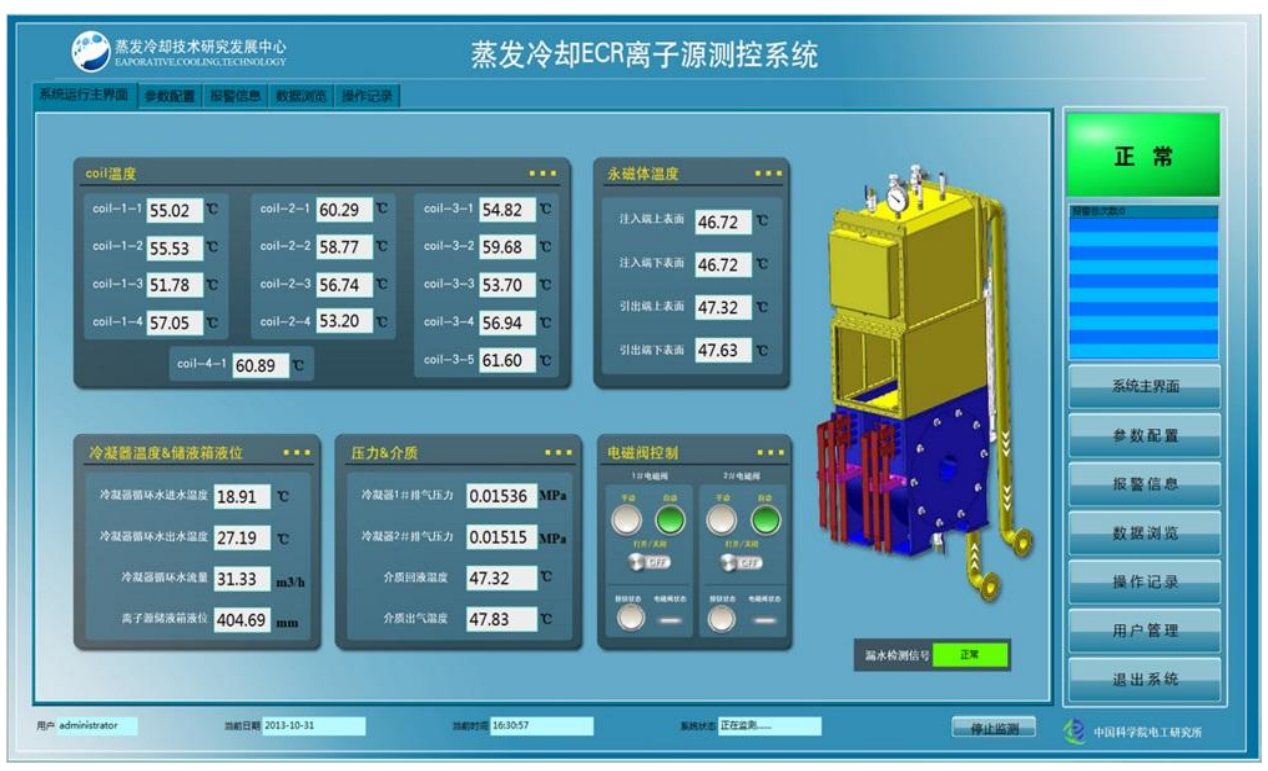

Fig. 3 the monitoring interface of parameter

After several experimental researches, Intermediate design scheme [5,6] and optimizing design, we final complete the installation of the ISC evaporative cooling ECRIS named LECR4 in IMP, the first 
room-temperature evaporative cooling ECRIS in the world. Fig.3 is the monitoring interface of evaporative cooling system showing the solenoid and magnet temperatures and second cooling water parameters. The on-site testing showed that the surface temperature of solenoid coils is almost around $60^{\circ} \mathrm{C}$, the temperature of the sextupole permanent magnet is about $47^{\circ} \mathrm{C}$ not exceeding $50^{\circ} \mathrm{C}$. The cooling target was reached and the operation is very stable with nearly no need of maintenance for the cooling system.

\section{Summary}

From analysis of physical and chemical characteristics,heat-transfer model experiment, instrumental inspection to final demonstrating application in LECR4, the coolant has been proved to be a good choice for the cooling of ECRIS. The evaporative cooling system with this coolant is simple, the structure is compact and the operation is safe and reliable. Which shows the application of the evaporative cooling technology to the ECRIS is successful and this kind of high efficient cooling mode can be extended to use.

\section{Acknowledgements}

This research was financially supported by the large scientific facility research project of Chinese Academy of Sciences. Thanks to IMP for providing support for on-site testing.

\section{References}

[1] Ruan Lin, The basic theory research of the inner evaporative cooling system for the large hydrogenerator and the simulation of the CLSC system [D], The Chinese Academy of Sciences, Beijing. (2004).

[2] Yang Shiming, Heat Transfer, second ed., Heigher Education Press, Beijing, 1987.

[3] Lin Ruan, Gu Guobiao, et al, The comparison of cooling effect between evaporative cooling method and inner water cooling method for the large hydro generator, Proceedings of the 11th International Conference on Electrical Machines and Systems (2007) 989-992.

[4] Ruan Lin, a kind of evaporative cooling equipment for ECR ion source, China. Patent ZL 20102 0244325.1. (2011).

[5] W. Lu, etc, DRAGON: A NEW 18 GHz RT ECRIS WITH A LARGE PLASMA CHAMBER, Proceedings of ECRIS(2010) 58-60.

[6] W.lu, etc, Development of DRAGON electron cyclotron resonance ion source at Institute of Modern Physics, review of Scientific Instruments. 83, 02 A328 (2012). 